\title{
Effects of ethanol withdrawal on the activity of rho-kinase in rat brain
}

\author{
Kurt $\mathrm{AH}^{1}$, Macit $\mathrm{E}^{2}$, Uzbay $\mathrm{T}^{3}$, Buyukafsar $\mathrm{K}^{4}$ \\ Department of Pharmacology, Medical Faculty, Kahramanmaras Sutcu Imam University, \\ Kahramanmaras, Turkey. farma1975@hotmail.com
}

\begin{abstract}
Besides its effect regarding addiction, ethanol also damages the central nervous system when it is used at high doses for a long time. The increase in the activity of Rho/Rho kinase pathway leads to central nervous system pathologies such as cerebral injury and epileptogenesis. The aim of this study was to investigate the contribution of Rho/Rho Kinase pathway to the degenerative and addictive effects of ethanol. For this purpose, we determined the Rho-kinase activity in striatum and hippocampus of rat brain. Wistar rats were treated with ethanol in a special liquid diet for 21 days. An isocaloric liquid diet without ethanol was given to the rats in the control group during the study. At the end of the 21 day ethanol exposure, one group was kept on taking ethanol and another group was withdrawn from ethanol. The rats were decapitated and their brains were taken out. Striatum and hippocampus were isolated. Phospho-moesin protein levels were measured in striatum and hippocampus homogenates using by Western blot analysis. The Rho-kinase (ROCK) activity in the striatum was found to be significantly decreased in ethanol exposed rats. In the hippocampus, there was a significant increase in the ROCK activity in the ethanol group. Our results indicated that ethanol caused some significant changes in Rho/ Rho Kinase pathway in rat brain (Fig. 2, Ref. 25). Text in PDF www.elis.sk.

KEY WORDS: ethanol, Rho-kinase, hippocampus, striatum, rat.
\end{abstract}

\section{Introduction}

Ethanol abuse and dependence remain among the most common substance abuse problems worldwide. Ethanol is a poison with direct toxic effects on nerve and muscle cells. Alcohol-related neurologic diseases include Wernicke-Korsakoff disease, alcoholic cerebellar degeneration, alcoholic myopathy, alcoholic neuropathy, foetal alcohol syndrome, alcohol withdrawal syndrome with seizures and delirium tremens. The discontinuation of chronic administration of ethanol is associated with excitatory withdrawal signs called ethanol withdrawal syndrome. The signs of ethanol withdrawal syndrome in humans (Thompson, 1978) and rodents (Majchrowicz, 1975) have been well determined the mechanisms underlying physical dependence to ethanol and ethanol withdrawal syndrome are poorly understood.

Rho-kinase (ROCK) contributes to several physiological processes by modulating cell migration, proliferation and survival, axonal outgrowth, dendrogenesis, endocytosis and tumour metas-

${ }^{1}$ Department of Pharmacology, Medical Faculty, Kahramanmaras Sutcu Imam University, Kahramanmaras, Turkey, ${ }^{2}$ Department of Pharmacology, Medical Faculty, Gulhane Military Medical Academy, Ankara, Turkey, ${ }^{3}$ Neuropsychopharmacology Application and Research Center, Uskudar University, Istanbul, Turkey, and ${ }^{4}$ Department of Pharmacology, Medical Faculty, Mersin University, Mersin, Turkey

Address for correspondence: A.H. Kurt, MD, Kahramanmaras Sutcu Imam University, Faculty of Medicine, Department of Pharmacology, 46050 Kahramanmaras, Turkey.

Phone: +90.344.2237666, Fax: +90.344.2257534 tasis. Abnormal activation of the Rho/ROCK pathway has been observed in various disorders of the central nervous system (CNS) (Mueller et al, 2005), and it is important in the pathogenesis of several neurological diseases such as spinal cord injury, stroke, multiple sclerosis and neuropathic pain (Mueller et al, 2005).

The inhibition of ROCK by the ROCK inhibitors improved mechanical hypoalgesia and motor dysfunction caused by spinal canal stenosis (Ito et al, 2007), protected against ischemia-induced delayed neuronal death when treatment was started $24 \mathrm{~h}$ after ischemia (Satoh et al, 2008), decreased tumour cell metastasis and invasion (Kamai et al, 2003; Lepley et al, 2005). The Rho family proteins RhoA, Rac1, and Cdc42 have been implicated in the phosphorylation of moesin in various cell lines. The activation of RhoA, Rac1, or Cdc42 is involved in the phosphorylation of moesin. In neuronal cells, moesin is highly expressed in PC12 cells and glioma cells, whereas the three ERM proteins are equally expressed in astrocytes (Songhee et al, 2008).

The aim of the present study was to investigate the contribution of Rho/Rho-kinase pathway to ethanol withdrawal. The regions of the brain named as striatum and hippocampus are related with ethanol addiction and withdrawal. We measured the amount of $\mathrm{p}$ moesin as an indicator of the Rho-kinase activity in these regions.

\section{Methods}

Animals and laboratory

All procedures in this study are in accordance with the Guide for the Care and Use of Laboratory Animals as adopted by the 
National Institutes of Health. Local ethical committee approval was also attained. All efforts were made to minimize animal suffering and to reduce the number of animals used. The experimental subjects were adult male Wistar rats (266-278 g weight at the beginning of the experiments). They were housed in a quiet and temperature and humidity-controlled room $\left(22 \pm 3{ }^{\circ} \mathrm{C}\right.$ and $65 \pm 5$ $\%$, respectively) in which a 12-h light/dark cycle was maintained (07:00-19:00 h light). Exposure to ethanol and all behavioral experiments involved in ethanol withdrawal syndrome were carried out in the separate and isolated laboratories, which have the same environmental conditions with the colony room.

\section{Chronic exposure to ethanol}

The rats were housed individually and ethanol was given in the modified liquid diet for chronic ethanol exposure as previously described (Uzbay et al, 1995). They received a modified liquid diet with or without ethanol ad libitum. No extra chow or water was supplied. The composition of the modified liquid diet with ethanol is as follows: cow's milk $925 \mathrm{ml}$ (Danone, Turkey), 25-75 ml ethanol (96.5\% ethyl alcohol; Tekel, Turkish State Monopoly), sucrose $17 \mathrm{~g}$. This mixture supplies $1000.7 \mathrm{kcal} / 1$. At the beginning of the study, rats were given the modified liquid diet without ethanol for 7 days. Then liquid diet with $2.4 \%$ ethanol has been administered for 3 days. The ethanol concentration was increased to $4.8 \%$ during the following 4 days and finally to 7.2 $\%$ for 21 days. Liquid diet was freshly prepared daily and presented at the same time of the day (10:00 h). The weight of the rats was recorded every day, and daily ethanol intake was measured and expressed as grams per kilograms per day. Control rats $(\mathrm{n}=$ 3) were pair fed an isocaloric liquid diet containing sucrose as a caloric substitute to ethanol.

\section{Evaluation of ethanol withdrawal syndrome}

At the end of the exposure to $7.2 \%$ ethanol-containing liquid diet, ethanol was removed from the diet. First group was kept on receiving ethanol and the other group was exposed to ethanol withdrawal.

\section{Western blot analysis}

All the rats which received ethanol, those exposed to ethanol withdrawal and exposed to an audiogenic stimulus were decapitated at 21 st days. Brains were removed and corpus striatum and hippocampus were dissected within 5 min on a block of ice. Corpus striatum and hippocampus was homogenized with lyses buffer solution, (composition: Tris- $\mathrm{HCl}$ (pH 7.4) $50 \mathrm{mM}, \mathrm{NaCl} 400 \mathrm{mM}$, EGTA $2 \mathrm{mM}$, EDTA $1 \mathrm{mM}$, dithiothreitol $1 \mathrm{mM}$, phenylmethylsulphonyl fluoride $10 \mu \mathrm{M}$, leupeptin $1 \mu \mathrm{g} / \mathrm{mL}$, pepstatin $1 \mu \mathrm{g} / \mathrm{mL}$, benzamidine $1 \mathrm{mM}$ ). The homogenate was centrifuged at 13000 $\mathrm{g}$ for $10 \mathrm{~min}$ at $4{ }^{\circ} \mathrm{C}$ and the supernatant was removed. It was then used for protein analysis (Bradford method) and Western blot analysis. Equal amounts of proteins $(50 \mu \mathrm{g})$ were loaded in wells, electrophoresed on $8 \%$ polyacryamide-SDS gels and then transferred to a nitrocellulose membrane overnight. The membrane was blocked with the blocking agent of an enhanced chemiluminescence kit (ECL plus; Amersham Biosciences, Freiburg, Germany) in Tris-buffered solution containing $0.05 \%$ Tween-20 for $1 \mathrm{~h}$. It was then probed with primary antibodies raised against $\mathrm{p}$-moesin (Polyclonal IgG, Santa Cruz Biotechnology Inc., CA, U.S.A.) at $1: 200$ dilution, followed by a horseradish peroxidase-conjugated secondary antibody (donkey anti-goat, $1: 1000$, Santa Cruz Biotechnology Inc.). Protein blots were then detected with the plus chemiluminescence detection kit (Amersham Biosciences) and visualized on commercial X-ray film.

\section{Statistical analysis}

All data represent the mean \pm standard error of the mean (S.E.M.) of n observations. For statistical comparison, one way analysis of variance (ANOVA) followed by the Bonferroni post hoc test or Student's t test, if appropriate, was used. The P value lower than 0.05 was considered significant.

\section{Results}

Phosphorylation of Moesin at Thr-558 in the Hippocampus and Striatum

In hippocampus, there was an increase in the ROCK activity in the group taking ethanol $(\mathrm{p}<0.05)$ and no change in the other group that was exposed to ethanol withdrawal (Fig. 1). The ROCK activity in the striatum decreased significantly $(p<0.01)$ in the

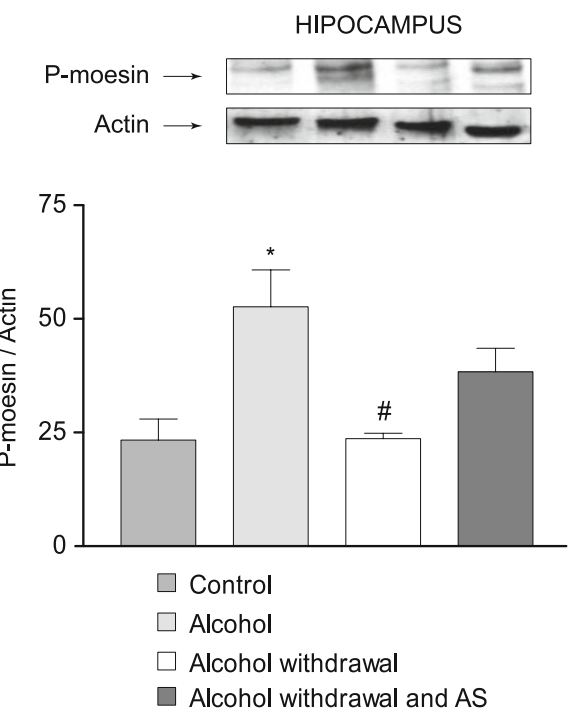

Fig. 1. Hippocampal p-moesin protein expression was determined by Western blotting. The homogenates were submitted to sodium dodecyl sulphate (SDS)-PAGE with $8 \%$ polyacrylamide and then transferred to a nitrocellulose membrane $(0.22 \mu \mathrm{m}$, Santa Cruz). The membrane was blocked with the blocking agent of the enhanced chemiluminescence advance kit (ECL plus) in Tris-buffered solution containing 0.05 $\%$ Tween- 20 (TBS-T) for $1 \mathrm{~h}$. It was then probed with a primary antibody raised against p-moesin (Polyclonal IgG, Santa Cruz) at 1 : 200 dilution, followed by horseradish peroxidase-conjugated secondary antibody (donkey antigoat, $1: 1000$ ). Actin was used as loading control. Blots were then assayed with an Enhanced Chemiluminescence Detection Kit (ECL plus, Amersham Bioscience), and visualized on commercially available $X$-ray films (* vs control; $p<0.05$, \# vs alcohol; $\mathrm{p}<\mathbf{0 . 0 5}$ ). 


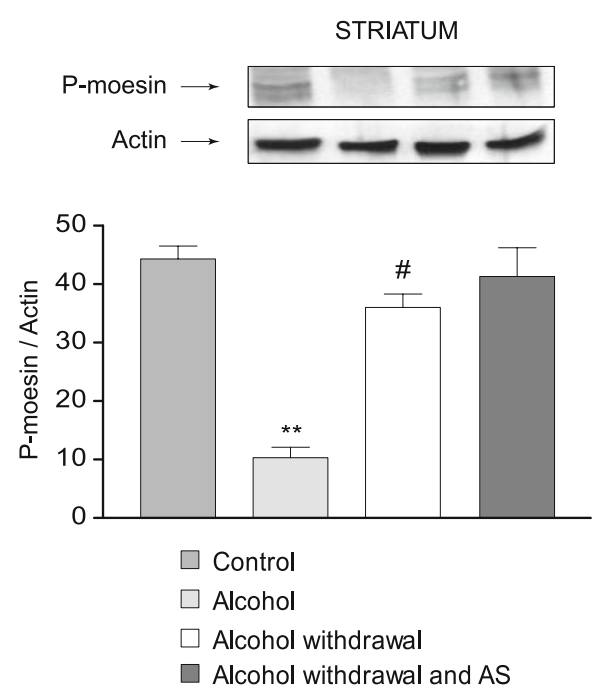

Fig. 2. Striatal p-moesin protein expression was determined by Western blotting. The homogenates were submitted to sodium dodecyl sulphate (SDS)-PAGE with $8 \%$ polyacrylamide and then transferred to a nitrocellulose membrane $(0.22 \mu \mathrm{m}$, Santa Cruz). The membrane was blocked with the blocking agent of the enhanced chemiluminescence advance kit (ECL plus) in Tris-buffered solution containing $0.05 \%$ Tween- 20 (TBS-T) for $1 \mathrm{~h}$. It was then probed with a primary antibody raised against p-moesin (Polyclonal IgG, Santa Cruz) at $1: 200$ dilution, followed by horseradish peroxidase-conjugated secondary antibody (donkey antigoat, $1: 1000$ ). Actin was used as loading control. Blots were then assayed with an Enhanced Chemiluminescence Detection Kit (ECL plus, Amersham Bioscience), and visualized on commercially available $X$-ray films $(* *$ vs control; $p<0.01$, \# vs alcohol; $\mathbf{p}<\mathbf{0 . 0 5}$ ).

group taking ethanol and increased $(\mathrm{p}<0.05)$ in another group where ethanol was removed (Fig. 2).

\section{Discussion}

This is the first study that determines the connection between alcohol withdrawal and Rho/Rho-kinase pathway. The main result obtained from the present study was that the ROCK activity in the striatum decreased significantly in the group taking ethanol and increased in the group that ethanol was removed. In hippocampus, there was an increase in the ROCK activity in the group taking ethanol and no change in the group that was exposed to ethanol withdrawal. These changes in the ROCK activity may play a role in the symptoms caused by ethanol withdrawal. Dopamin and serotonin may be responsible for the changes in the ROCK activity. Recent studies determined that haloperidol, a nonspecific dopaminergic antagonist, increased stereotyped behavior during the same period of ethanol withdrawal in rats (Uzbay et al, 1994). In addition, it was seen that serotonin re-uptake inhibitors lowered the symptoms.

Synaptic plasticity is dependent on the structural regulation of the actin cytoskeleton in dendritic spines (Carlisle et al, 2005; Schuber et al, 2007; Sekino et al, 2007). Sekino et al (2007) and Schubert and Dotti (2007) have reviewed in detail the molecu- lar players involved in the maintenance of the synaptic cytoarchitecture in dendritic spines, such as the regulation of F-actin cytoskeleton through the various actin-binding proteins and the molecular regulators of actin dynamics, i.e. membrane receptors and downstream signalling cascades. Rho GTPases have emerged as the key regulators in the control of actin filament assembly in the dendritic spines (Schuber et al, 2007; Sekino et al, 2007). The Rho family GTPases including RhoA, Rac1, and CDC42 are the major regulators in synaptic plasticity, both in dendrite morphogenesis and stability as well as in growth cone motility and collapse (Lin et al, 2007). Small Rho proteins act as molecular switches between inactive GDP-bound and active GTP-bound forms under the regulation of several Rho GEFs (guanine nucleotide exchange factors) and Rho GAPs (GTPase activating protein) (Linseman et al, 2008; Schiller, 2006). In neurons, there are several upstream regulators of Rho GTPases, such as NMDA, ephrin-B, and semaphorin $3 \mathrm{~A}$ receptors as well as a number of Rho GEFs, which can activate the Rho proteins and in that way subsequently modulate several downstream effector proteins (Lim et al, 2008; Kasri et al, 2008) Kalirin-7, and Tiam1 are well-known Rho GEFs, which can regulate the morphogenesis of dendritic spines. RhoA, Rac1, and Cdc42 proteins are linked to distinct upstream and downstream regulators. In many instances, RhoA, and Rac1/Cdc42 have antagonistic effects on dendritic plasticity, this being mostly due to their different effector molecules. RhoA-GTP activates the Rhokinases (ROCK1, and ROCK2) and ROCK2 is highly expressed in the brain.

To evaluate the in vivo functions of CNS ROCKs, inhibitors have been administered in several model systems. Beneficial neurological effects were observed in animal models of Alzheimer's disease, neuropathic pain, demyelinating/inflammatory diseases, stroke and spinal-cord injuries (Bito et al, 2000; Zhou et al, 2003; Eldawoody et al, 2010). Further analysis of these pathways is likely to accelerate molecular understanding in neuroscience and provide the potential for novel therapeutics in neurology.

Glutamatergic system and NMDA receptors have been proposed to have a crucial role in the development of the ethanol withdrawal signs (Krystal et al, 2003). Glutamate is an excitatory neurotransmitter, which is responsible for brain damage and also for epileptogenesis, activates Rho-kinase in neuronal cells (Jeon et al, 2002). Furthermore, it has been recently demonstrated that the Rho/Rho-kinase signalling pathway is upregulated and may be involved in NMDA-induced retinal neurotoxicity, because fasudil, a Rho-kinase inhibitor, was found to be neuroprotective against glutamate-related excitotoxicity (Kitaoka et al, 2004). NMDA leads to an increase in retinal RhoA protein level and activates Rho-kinase, causing neuronal toxicity. Inhibition of Rho-kinase prevented glutamate induced neurotoxicity. Moreover, in cultured hippocampal neurons as well as in whole-brain synaptosomal fractions, RhoA was associated with glutamate receptors at the plasma membrane of dendritic spines (Schubert et al, 2006). These findings, although not directly linking ROCK action as the underlying mechanism for neuron damage, are suggestive of this connection since the inhibition of ROCK may be involved in ethanol withdrawal syndrome. 
In conclusion, our results suggest that Rho-kinase activity in some areas of rat brain is influenced by ethanol consumption and withdrawal. The drugs that affect on brain Rho-kinase system may be effective in the treatment of alcohol withdrawal syndrome.

\section{References}

1. Thompson WL. Management of alcohol withdrawal syndromes. Adv Intern Med 1978; 138: 278-283.

2. Majchrowicz E. Induction of physical dependence upon ethanol and the associated behavioral changes in rats. Psychopharmacol 1975; 43: 1993-1996.

3. Mueller BK, Mack H, Teusch N. Rho kinase, a promising drug target for neurological disorders. Nat Rev Drug Discov 2005; 4: 387-398.

4. Ito T, Ohtori S, Hata K, Inoue G, Moriya H. Rho kinase inhibitor improves motor dysfunction and hypoalgesia in a rat model of lumbar spinal canal stenosis. Spine 2007; 32: 2070-2075.

5. Satoh S, Toshima Y, Hitomi A, Ikegaki I, Seto M. Wide therapeutic time window for Rho-kinase inhibition therapy in ischemic brain damage in a rat cerebral thrombosis model. Brain Res 2008; 1193: 102-108

6. Kamai T, Tsujii T, Arai K, Takagi K, Asami H. Significant association of Rho/ROCK pathway with invasion and metastasis of bladder cancer. Clin Cancer Res 2003; 39: 2632-2641.

7. Lepley D, Paik JH, Hla T, Ferrer F. The G protein-coupled receptor $\mathrm{S} 1 \mathrm{P} 2$ regulates Rho/Rho kinase pathway to inhibit tumor cell migration. Cancer Res 2005; 65: 3788-3795.

8. Songhee J, Sohee K, Jong-Bae P, Ghill Suh K, Chang-Dae B. RhoA and Rho Kinase-dependent Phosphorylation of Moesin at Thr-558 in Hippocampal Neuronal Cells by Glutamate. J Biological Chemıstry 2002; 19: 16576-16584.

9. Uzbay IT, Akarsu ES, Kayaalp SO. Effects of flumazenil on ethanol withdrawal syndrome in rats. Arzneim Forsch-Drug Res 1995; 45: 120-124.

10. Uzbay IT, Akarsu ES, Kayaalp SO. Effects of bromocriptine and haloperidol on ethanol withdrawal syndrome inrats. Pharmacol Biochem Behav 1994; 49: 969-974.

11. Carlisle HJ, Kennedy MB. Spine architecture and synaptic plasticity. Trends Neurosci 2005; 28: 182-187.

12. Schuber V, Dotti CG. Transmitting on actin: synaptic control of dendritic architecture. J Cell Sci 2007; 120: 205-212.
13. Sekino Y, Kojima N, Shirao T. Role of actin cytoskeleton in dendritic spine morphogenesis. Neurochem Int 2007; 51: 92-104.

14. Lin X, Ogiya M, Takahara M, Yamaguchi W, Furuyama T. Sema4D-plexin-B1 implicated in regulation of dendritic spine density through RhoA/ROCK pathway. Neurosci Lett 2007; 428: 1-6.

15. Linseman A, Loucks A. Diverse roles of Rho family GTPases in neuronal development, survival, and death. Front Biosci 2008; 13: 657-676.

16. Schiller M. Coupling receptor tyrosine kinases to Rho GTPases - GEFs what's the link. Cell Signal 2006; 18: 1834-1843.

17. Lim K, Matsuda N, Poo M. Ephrin-B reverse signaling promotes structural and functional synaptic maturation in vivo. Nature Neurosci 2008; 11: 160-169.

18. Kasri N, Van-Aelst L. Rho-linked genes and neurological disorders, Pflugers Arch Eur J Physiol 2008; 455: 787-797.

19. Bito H, Furuyashiki T, Ishihara H, Shibasaki Y, Ohashi K. A critical role for a Rho-associated kinase, p160ROCK, in determining axon outgrowth in mammalian CNS neurons. Neuron 2000; 26: 431-441.

20. Zhou Y, Su Y, Li B, Liu F, Ryder JW. Nonsteroidal antiinflammatory drugs can lower amyloidogenic Abeta 42 by inhibiting Rho. Science 2003; 302: 1215-1217.

21. Eldawoody H, Shimizu H, Kimura N, Saito A, Nakayama T. Fasudil, a Rho-kinase inhibitor, attenuates induction and progression of cerebral aneurysms: experimental study in rats using vascular corrosion casts. Neurosci Lett 2010; 5: 76-80.

22. Krystal JH, Petrakis IL, Mason G, Trevisan L, D'Souza DC. Nmethyl-D-aspartate glutamate receptors and alcoholism: reward, dependence, treatment, and vulnerability. Pharmacol Ther 2003; 99: 79-94.

23. Jeon S, Kim S, Park JB, Suh PG, Kim YS. RhoA and Rho kinasedependent phosphorylation of moesin at Thr-558 in hippocampal neuronal cells by glutamate. J Biol Chem 2002; 277: 16576-16584.

24. Kitaoka Y, Kumai T, Lam TT, Kuribayashi K, Isenoumi K. Involvement of RhoA and possible neuroprotective effect of fasudil, a Rho kinase inhibitor, in NMDA-induced neurotoxicity in the rat retina. Brain Res 2004; 1018: 111-118.

25. Schubert V, Da Silva JS, Dotti CG. Localized recruitment and activation of RhoA underlies dendritic spine morphology in a glutamate receptor-dependent manner. J Cell Biol 2006; 172: 453-467.

Received August 9, 2014. Accepted March 8, 2015. 